\title{
Intervención en poblaciones de riesgo (1): los hijos de alcohólicos
}

\author{
DíAz, R. '; FerRI, M ${ }^{\text {a J. }}{ }^{2}$ \\ (1) Coordinadora del Programa ALFIL. Socidrogalcohol. Unidad de Alcohología de la Generalitat de Catalunya (Hospital Clínic de Barcelona). \\ (2) Grupo ALFIL. Socidrogalcohol. Unidad de Alcohología de Alcoi (Alicante).
}

\section{RESUMEN}

Se revisan los factores de riesgo y de resistencia en los hijos de alcohólicos, así como la metodología óptima para la intervención preventiva en este grupo de alto riesgo para el alcoholismo y otros trastornos relacionados. Se propone un plan integral de intervención que incluiría la detección precoz de casos, la motivación de las familias para participar en los programas, los grupos educativos para padres y para hijos sobre temas relacionados con el alcoholismo en la familia y, por último, actividades terapéuticas más intensas dirigidas a los casos de mayor riesgo.

Se describe también la experiencia del Programa ALFIL en la realización de grupos psicoeducativos para hijos de alcohólicos y para sus padres, en el marco de centros de tratamiento del alcoholismo y asociaciones de alcohólicos rehabilitados de diversas comunidades españolas. Los resultados de la evaluación de 13 grupos realizados entre 1998 y 2000 indican que este tipo de actividades preventivas son valoradas satisfactoriamente por los participantes, y tienen resultados positivos relacionados principalmente con un mayor conocimiento sobre el alcohol por parte de los jóvenes, y con la percepción subjetiva de más diálogo y menos conflictos familiares tras las sesiones en un porcentaje significativo de los participantes.

Se concluye que, en los próximos años, la investigación en este campo deberá dirigirse hacia las técnicas de detección precoz, la evaluación sistemática de factores de riesgo y protección, las estrategias motivacionales específicas para esta población y la evaluación continuada de experiencias preventivas. Se constata, asimismo, la necesidad de continuar trabajando en el diseño de materiales preventivos y de autoayuda para estas familias, en la formación de profesionales y en la diseminación de este tipo de intervenciones en nuestro entorno socio-cultural.

Palabras clave: hijos de alcohólicos, prevención, grupos, familia.

\begin{abstract}
This chapter reviews risk and resilience factors in children of alcoholics and the ideal methodology for preventive interventions in this high risk group for alcoholism and other related psychopathologies. An integral intervention plan is proposed including early case detection, family motivation to take part in the programme, and educational groups for parents and for children about topics related to alcoholism in the family. Also, in depth therapeutic interventions addressing very high risk cases are proposed.

The ALFIL Program experience in psychoeducational groups for children of alcoholics and their parents have been conducted in several treatment centres for alcoholism and associations of recovered alcoholics in Spain. Evaluation results of 13 group interventions from 1998 to 2000 indicate that this kind of educational activities are positively evaluated by participants, both parents and children, and have positive results like a significant increase in alcohol knowledge about alcohol in children, and a subjective perception of increased dialogue and decreased conflict in the family after participating in group sessions in a significant percentage of participants.

It is concluded that, in the following years, research in this field should be addressed to early detection techniques, systematic evaluation of risk and resilience factors, specific motivational strategies and the continuous evaluation of preventive interventions. It is also stated the need of designing specific preventive and self-help materials for alcoholic families, professional training, and dissemination of this kind of interventions in our country.
\end{abstract}

Key words: children of alcoholics, prevention, groups, family. 


\section{LOS HIJOS DE ALCOHÓLICOS: UNA PO- BLACIÓN VULNERABLE}

Los hijos de alcohólicos, alrededor del $10 \%$ de la población según algunas estimaciones ${ }^{1-3}$, constituyen un grupo de la población con una elevada probabilidad de desarrollar problemas diversos como consecuencia de interacciones complejas entre tres tipos de factores biopsico-sociales"

1) Los efectos ambientales de la convivencia con progenitores alcohólicos ${ }^{5}$

2) La transmisión genética de factores de riesgo relacionados con el alcoholismo $6,7,8$

3) Los efectos tóxicos del alcohol sobre las células germinales y sobre los órganos en desarrollo del feto ${ }^{9,10}$

Los efectos adversos del alcoholismo paterno que han sido descritos con mayor frecuencia están relacionados con abusos físicos y psicológicos, signos y síntomas de alcoholización fetal, enfermedades psicosomáticas, trastornos cognitivos y de personalidad, problemas de conducta, fracaso escolar, depresión, ansiedad, alcoholismo y otras drogadicciones. ${ }^{11-21}$

También resulta frecuente que los hijos de alcohólicos acaben adoptando patrones cognitivos y de comportamiento disfuncionales en un intento de adaptarse a su ambiente familiar desestructurado. Se han descrito algunos patrones típicos como el "héroe o salvador", el "niño problemático" o "chivo expiatorio", el "niño perdido u olvidado" y el "payasete o mascota de la familia". ${ }^{22,23}$ Si no se corrigen a tiempo, estos patrones pueden convertirse, en la vida adulta, en verdaderos trastornos de personalidad como el obsesivocompulsivo, el antisocial, el de evitación, el límite o el co-dependiente. ${ }^{24}$

Sin embargo, no todos los hijos de alcohólicos desarrollan problemas severos. En realidad, los efectos del alcoholismo paterno son muy variables, dependiendo de la interacción entre los factores de riesgo y los factores de resistencia que se encuentran presentes en cada caso concreto. ${ }^{25,26}$

\section{MODELOS DE VULNERABILIDAD. RIES- GOY RESISTENCIA}

En las últimas décadas se han formulado diversos modelos teóricos para tratar de explicar la naturaleza compleja y multifactorial del riesgo y también la heterogeneidad en las respuestas de los hijos de alcohólicos. 27-29,26,8,30,31,7

Uno de los modelos de vulnerabilidad más completo y potencialmente más útil para guiar las intervenciones preventivas específicas para hijos de alcohólicos es el modelo de Sher ${ }^{26}$, un modelo holístico o globalizador que sugiere que la historia familiar de alcoholismo podría conducir al desarrollo de diversos problemas, a través de diferentes vías. Más concretamente, el modelo propone tres vías principales a través de las cuales los hijos de alcohólicos pueden acabar desarrollando el mismo trastorno que sus progenitores: (1) la vía del afecto negativo o malestar emocional, (2) la vía de la desviación social y (3) la vía del refuerzo aumentado debido a una mayor sensibilidad a los efectos reforzadores del alcohol (Figura 1).

Numerosos estudios han confirmado que los hijos de alcohólicos presentan algunas características bio-psico-sociales diferenciales que podrían constituir "marcadores de riesgo" para el desarrollo de alcoholismo u otros trastornos relacionados. ${ }^{6}$ Algunos de estos marcadores han mostrado capacidad predictiva sobre el desarrollo de problemas relacionados con el alcohol u otras drogas, ${ }^{32-35}$ lo que les confiere utilidad potencial para el screening o detección precoz de sujetos especialmente vulnerables, con objeto de dirigirlos precozmente a programas preventivos ${ }^{36-39}$ (véase la columna izquierda de la Tabla 1).

Profundizar en el conocimiento de los factores de riesgo característicos en los hijos de alcohólicos podría ser muy útil para desarrollar estrategias de screening y detección pre$\mathrm{coz}$ de sujetos vulnerables. Sin embargo, cuando se trata de diseñar intervenciones preventivas específicas en este sector de la población, nos encontramos con el hecho de que algunos de los factores de riesgo más 
Figura 1. Modelo de vulnerabilidad para el alcoholismo familiar: (1) Vía del afecto negativo, (2) Vía de la desviación social, (3) Vía del refuerzo aumentado. Modelo simplificado, modelo de Sher ${ }^{26}$.

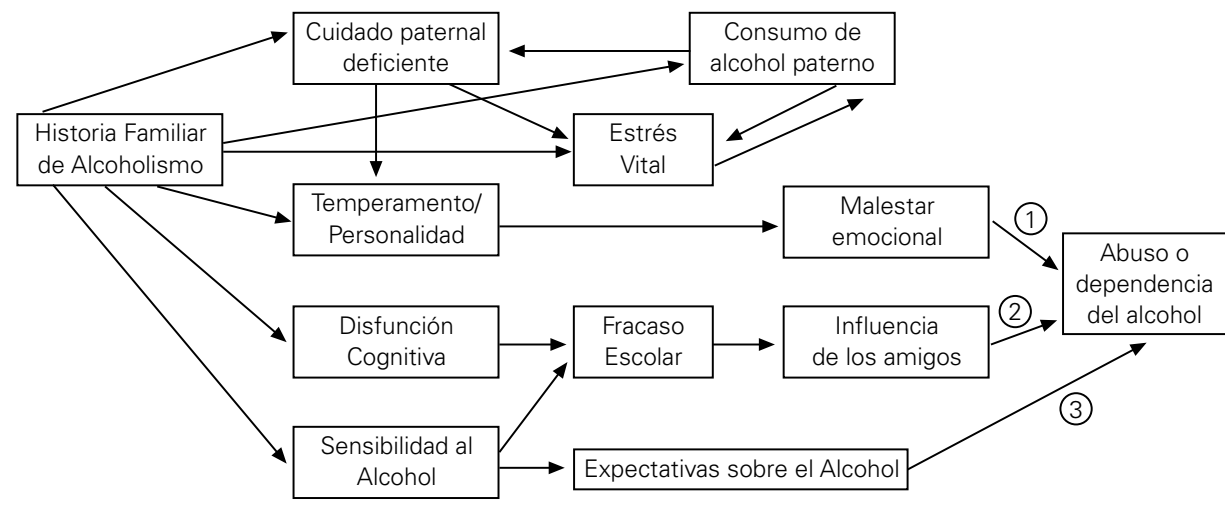

comunes (e.g. el funcionamiento hiperreactivo del sistema nervioso o la sensibilidad diferencial al alcohol u otras drogas) resultan muy difíciles o imposibles de modificar directamente. Por ello, en las intervenciones dirigidas a éste y a otros colectivos de riesgo afines, se suele hacer un énfasis especial en intentar potenciar los factores de protección o de resistencia que pueden contrarrestar o compensar el efecto de los factores de riesgo..$^{40}$ En la columna derecha de la Tabla 1 se ha intentado resumir aquellos factores que han mostrado un efecto protector ante posibles consecuencias negativas en los hijos de alcohólicos en diversos estudios. . $^{41-43,38,44}$

\section{3. ¿CÓMO SE DEBE INTERVENIR?}

Tras varias décadas de debate en los EE.UU. entre los seguidores y los detractores del Movimiento COAs (Children of Alcoholics), los primeros partidarios del intervencionismo y los segundos contrarios a la intervención indiscriminada en este sector de la población, los expertos han llegado a un consenso acerca de la necesidad de intervención. ${ }^{45,46,63}$ En nuestros días, la mayor parte de los autores ofrecen algunos de los siguientes argumentos a favor de las intervenciones preventivas selectivas para los jóvenes afectados por problemas de alcohol en su familia. En primer lugar, existen casos de riesgo extremado que requieren una detección precoz y una actuación terapéutica rápida y/o intensa, como los niños que son víctimas de la alcoholización fetal, de abusos físicos o sexuales, de negligencia en el cuidado paterno, etc. Por otro lado, aún aceptando que, en general, los efectos negativos que sufren los hijos de alcohólicos no son uniformes ni específicos, ${ }^{24,19}$ algunos estudios han mostrado que este colectivo podría presentar factores de riesgo premórbidos característicos que podrían requerir un abordaje preventivo específico lo más precoz posible. . $^{6,4,48}$

Otro argumento a favor de la intervención selectiva dirigida a este sector de la población sería que las familias alcohólicas presentan frecuentemente mecanismos de negación y ocultación de los problemas, dificultades importantes para hablar del "secreto familiar" y sentimientos intensos de culpa y vergüenza que dificultan su acceso espontáneo a los programas existentes y que requieren un abordaje motivacional específico. ${ }^{49}$ 
Tabla 1. Factores de riesgo y factores de protección en los hijos de alcohólicos.

\begin{tabular}{|c|c|c|}
\hline \multicolumn{3}{|c|}{ HIJOS DEALCOHÓLICOS } \\
\hline & FACTORES DE RIESGO & FACTORES DE PROTECCIÓN \\
\hline : & $\begin{array}{l}\text { Antecedentes familiares de alcoholismo y otras psico- } \\
\text { patologías (número y parentesco) } \\
\text { Funcionamiento bioquímico y psicofisiológico particu- } \\
\text { lar del sistema nervioso (hipereactividad y dificultades } \\
\text { en la atención sostenida) } \\
\text { Disfunción neuropsicológica (frontal) } \\
\text { Rasgos de temperamento y personalidad vulnerable } \\
\text { (hiperactividad, conductas disociales, timidez, etc.) } \\
\text { Mayor sensibilidad a los efectos reforzadores del alco- } \\
\text { hol y/u otras drogas } \\
\text { Exposición al alcohol/otras drogas in utero }\end{array}$ & $\begin{array}{l}\text { Valores saludables y pensamiento prosocial } \\
\text { Autoaceptación y autosuperación } \\
\text { Expectativas de futuro positivas } \\
\text { Buen rendimiento intelectual } \\
\text { Pensamiento crítico e independiente } \\
\text { Sentido del humor } \\
\text { Locus de control interno y sentido de la responsabili- } \\
\text { dad } \\
\text { Estrategias de afrontamiento (emocionales y cogniti- } \\
\text { vas) } \\
\text { Flexibilidad, adaptabilidad, insight, autocontrol } \\
\text { Conocimientos básicos sobre el alcohol/otras drogas } \\
\text { Actitudes negativas y expectativas realistas respecto al } \\
\text { consumo de drogas }\end{array}$ \\
\hline 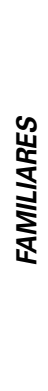 & $\begin{array}{l}\text { Negligencia en el cuidado paternal, abusos físicos o } \\
\text { psicológicos, falta de afecto } \\
\text { Gran número de acontecimientos vitales estresantes } \\
\text { Pautas educativas alteradas (ausencia de normas, } \\
\text { supervisión y refuerzos consistentes) } \\
\text { Falta de cohesión y comunicación, interrupción de los } \\
\text { rituales familiares, alto nivel de conflictos familiares } \\
\text { Dificultades económicas y/o culturales } \\
\text { Contacto precoz con el alcohol u otras drogas (imita- } \\
\text { ción de patrones de consumo y transmisión de actitu- } \\
\text { des favorables hacia el consumo) }\end{array}$ & $\begin{array}{l}\text { Vínculos familiares positivos y apoyo emocional } \\
\text { Sistema familiar estructurado, normativo y coherente } \\
\text { Progenitor no-alcohólico emocionalmente estable, } \\
\text { afectivo y acogedor } \\
\text { Estabilidad y cohesión familiar } \\
\text { Núcleo familiar pequeño (menos de } 4 \text { hijos) } \\
\text { Supervisión paterna adecuada } \\
\text { Mantenimiento de los rituales y celebraciones familia- } \\
\text { res } \\
\text { Comunicación padres-hijos abierta y sincera }\end{array}$ \\
\hline 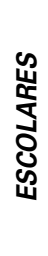 & $\begin{array}{l}\text { Dificultades en la relación con los compañeros o los } \\
\text { profesores (problemas de comportamiento) } \\
\text { Dificultades de aprendizaje y bajo rendimiento } \\
\text { Ausencia de vínculos significativos en la escuela } \\
\text { Fracaso y/o abandono escolar }\end{array}$ & $\begin{array}{l}\text { Vínculos con la escuela, los compañeros y los maes- } \\
\text { tros } \\
\text { Comunicación padres-escuela } \\
\text { Normas claras sobre las conductas aceptables } \\
\text { Supervisión y refuerzos coherentes con las normas } \\
\text { Expectativas de los maestros claras y adaptadas a las } \\
\text { capacidades del niño }\end{array}$ \\
\hline 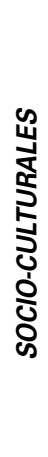 & $\begin{array}{l}\text { Presión del grupo de amigos y de la publicidad hacia } \\
\text { el consumo de alcohol/otras drogas } \\
\text { Costumbres y modas culturales (ej: mitos de las cultu- } \\
\text { ras vitivinícolas o implantación actual de patrones de } \\
\text { consumo anglosajón) } \\
\text { Disponibilidad de alcohol u otras drogas (bajo precio, } \\
\text { acceso fácil en casa, supermercados, etc.) } \\
\text { Situaciones extremas de pobreza, desarraigo cultural } \\
\text { o marginación social }\end{array}$ & $\begin{array}{l}\text { Vinculación a grupos o instituciones pro-sociales (reli- } \\
\text { giosos, deportivos, culturales, etc.) } \\
\text { Relaciones positivas con adultos significativos: maes- } \\
\text { tros, familiares, vecinos, etc. } \\
\text { Modelos adultos y amigos no-consumidores de drogas } \\
\text { Participación en actividades de grupo positivas (excur- } \\
\text { siones, juegos, deportes, trabajos escolares, etc.) } \\
\text { Normas sociales claras respecto al no-consumo de } \\
\text { drogas } \\
\text { Acceso a servicios comunitarios flexibles y adaptables } \\
\text { a las necesidades de los usuarios } \\
\text { Oportunidades para participar en actividades sociales y } \\
\text { de ocio alternativas al consumo de drogas }\end{array}$ \\
\hline
\end{tabular}


Por otro lado, la intervención sobre la familia alcohólica no sólo está relacionada con efectos positivos sobre el desarrollo psicológico de los hijos, sino que permite mejorar la dinámica familiar en general y el pronóstico del tratamiento del progenitor alcohólico, debido a un aumento de la motivación y de la adherencia al tratamiento, y de la comprensión y la acogida del afectado por parte de los familiares.

Algunos autores han defendido las intervenciones preventivas selectivas para los hijos de alcohólicos como una estrategia útil para reducir el alto coste que ocasiona a posteriori el tratamiento de este colectivo en el sistema sanitario público. ${ }^{50,51}$ Asimismo, se ha argumentado que los programas preventivos dirigidos a los hijos de alcohólicos podrían ser más rentables, en la relación coste-beneficio, que los programas universales dirigidos a los jóvenes en general, o incluso que los programas selectivos dirigidos a otros colectivos de riesgo menos numerosos. ${ }^{52}$

Por lo tanto, hoy en día, los artículos especializados ya no tratan de si se debe intervenir o no, sino de cómo debemos intervenir de forma efectiva para reducir el riesgo y aumentar la resistencia de los hijos de alcohólicos, haciendo énfasis en aquéllos que presentan mayor probabilidad de desarrollar problemas, y evitando posibles efectos contraproducentes relacionados con el etiquetaje, la desresponsabilización y el autocumplimiento de la profecía. ${ }^{53,54,38,24,25}$

En la última década, en los EE.UU. se han diseñado y extendido varios programas que incluyen intervenciones específicas para hijos de alcohólicos o de otros drogodependientes, algunos de los cuales han demostrado su eficacia experimentalmente: el SMAAP - Stress Management and Alcohol Awareness Program, ${ }^{55,56}$ el STAR - Students Together and Resourceful, ${ }^{57,58}$ el SFP - Strengthening Families Program ${ }^{59}$, el CASPAR - Cambridge and Somerville Program for Alcoholism Rehabilitation $^{60}$ y el ESCAPE. ${ }^{61,62}$ También en la Comunidad Europea, los hijos de alcohólicos han empezado a constituir una preocupación para las instituciones encargadas de la salud pública y diversas organizaciones han manifestado la necesidad de poner en marcha y extender estrategias preventivas específicas. ${ }^{63}$

Actualmente, en los EE.UU, la mayoría de las intervenciones preventivas selectivas para hijos de alcohólicos y otros jóvenes de alto riesgo se realizan desde la escuela, procuran dirigirse a toda la familia y siguen un enfoque más psico-educativo que psico-terapéutico. Estas intervenciones pretenden reducir la prevalencia de problemas relacionados con el consumo de drogas en los hijos de alcohólicos y en otros jóvenes de alto riesgo a través de diferentes vías: 1) el incremento del conocimiento y el cambio de actitudes y expectativas respecto el consumo de alcohol y otras drogas, 2) el entrenamiento en habilidades sociales y en estrategias de afrontamiento, tanto cognitivas como emocionales y 3 ) la potenciación de los factores de protección familiares (normas claras y coherentes, comunicación, comprensión y entendimiento mútuo, resolución de conflictos, etc.). ${ }^{64}$

A partir de la revisión realizada, se propone que las intervenciones para hijos de alcohólicos deberían seguir un plan de intervención integral que incluiría los siguientes pasos: 1) identificación precoz de casos (con especial énfasis en los de mayor riesgo), 2) intervención motivacional para promover la participación de toda la familia en los programas preventivos, 3) evaluación de factores de riesgo y de resistencia (para adaptar la intervención de forma individualizada), 4) grupos psicoeducativos para padres y para hijos (en centros de tratamiento, en escuelas, en centros recreativos para jóvenes o en centros de asistencia socio-sanitaria) y, en caso necesario, 5) psicoterapia apropiada a las necesidades detectadas (individual, grupal o familiar, entrenamiento cognitivo-conductual, psicodinámica, etc.)

Algunos autores han sugerido que los programas selectivos para hijos de alcohólicos deben basarse en una serie de principios básicos para ser eficaces en sus objetivos preventivos. ${ }^{43,65}$ En los casos de mayor riesgo, la intervención temprana e intensiva (en ocasio- 
nes incluso antes del nacimiento) es crucial para un buen pronóstico. ${ }^{47}$ Sin embargo, en los casos de riesgo leve a moderado, sería suficiente participar en grupos psico-educativos selectivos para pre-adolescentes y adolescentes (9-16 años) y/o para sus padres, ${ }^{62,55,65}$ con el fin de prepararlos para afrontar el período crítico de la adolescencia. ${ }^{40}$

El trabajo educativo y/o terapéutico en grupo es fundamental en las familias alcohólicas, ya que reduce la sensación de aislamiento, la vergüenza, la culpa, y la negación del problema familiar. Además, promueve la identificación con iguales, el apoyo emocional mutuo, y la confianza en los demás. En el caso de los hijos, el grupo facilita, además, el entrenamiento en habilidades sociales como la comunicación asertiva y la resistencia a la presión social, aspectos clave en todos los programas de prevención de drogas. Para los padres, el grupo es un lugar idóneo para compartir sus preocupaciones e intercambiar estrategias educativas saludables para sus hijos.

En los programas educativos para hijos de alcohólicos no deben faltar temas esenciales como una información objetiva sobre el alcohol y el alcoholismo apropiada para cada edad, los factores de riesgo y protección, los roles disfuncionales y los conflictos típicos en las familias alcohólicas, entre otros. También es esencial incluir el aprendizaje de técnicas de afrontamiento al estrés y al malestar emocional, así como estrategias para aumentar la autoestima, la autoeficacia y la competencia social. Finalmente, el entrenamiento en la toma de decisiones, la resolución de problemas y el auto-control también puede ser muy útil en este colectivo.

Otro aspecto importante en las intervenciones preventivas para hijos de alcohólicos es evitar alarmar o angustiar a este colectivo sobre las posibles repercusiones negativas de tener un progenitor alcohólico, ya que esto puede tener efectos contrapreventivos como el del autocumplimiento de la profecía. ${ }^{53,54}$ También es importante enseñar a los jóvenes estrategias de afrontamiento al estigma social que supone ser etiquetado como "hijo de alcohólico", incluso en su entorno natural ( "Eres igual que tu padre..." "PobreciIlo... ¿Qué se puede esperar de él teniendo el padre que tiene?"... "De tal palo... "). Precisamente para evitar los efectos del etiquetaje, en los EE.UU. la mayoría de los programas selectivos dirigidos a hijos de alcohólicos suelen realizarse en las escuelas, en centros juveniles o en otros entornos naturales para los jóvenes, y utilizan con frecuencia procedimientos de autoselección. . 6,57

Por último, para superar el obstáculo que supone la falta de motivación de las familias con mayor riesgo para participar en los programas preventivos existentes, algunos autores han sugerido el uso de estrategias específicas como las de la entrevista motivacional, el uso contingente de refuerzos como subsidios familiares, premios, comida o regalos, las órdenes judiciales, etc. ${ }^{49,66,67}$ La sensibilización y la formación específica de los agentes preventivos que están en contacto diario con estas familias, para que puedan ejercer su función de detección precoz y derivación de casos, también podría ser una pieza clave para el éxito de este tipo de intervenciones.

\section{EL PROGRAMA ALFIL}

A raíz de la necesidad detectada en los últimos años en nuestro país de evaluar y optimizar las intervenciones dirigidas a la prevención y el tratamiento de las repercusiones del alcoholismo paterno sobre la descendencia, y teniendo como base teórica y empírica los antecedentes descritos en los apartados anteriores, profesionales de Socidrogalcohol diseñaron en 1996 el Programa ALFIL, un programa de prevención familiar selectiva, que ha sido subvencionado desde 1997 por la Delegación del Gobierno para el Plan Nacional sobre Drogas. Este programa ha sido aplicado por personal socio-sanitario especializado en centros de tratamiento del alcoholismo y en asociaciones de alcohólicos rehabilitados de varias comunidades españolas, y está dirigido a hijos de alcohólicos cuyos progeni- 
tores han sido identificados como dependientes del alcohol en un centro especializado. ${ }^{68,69}$

El Programa ALFIL fue diseñado para cumplir dos objetivos básicos:

a) Realizar un estudio descriptivo de las características de los hijos de alcohólicos de 7 a 17 años cuyos padres entran en contacto con un centro especializado, y compararlas con las de un grupo control de la población general sin antecedentes familiares de la enfermedad, con el fin de averiguar si los primeros constituyen realmente una población de alto riesgo en nuestro país, sobre qué factores de riesgo específicos sería preciso intervenir y qué factores de protección se deberían fomentar. ${ }^{70,71}$

b) Evaluar el proceso de implantación y la eficacia de una intervención psicoeducativa experimental en grupos de hijos de alcohólicos pre-adolescentes y adolescentes, con el fin de analizar las características óptimas y las dificultades para realizar este tipo de intervenciones en nuestro entorno socio-cultural.

En este trabajo describiremos exclusivamente los objetivos, la metodología y algunos resultados de la evaluación de los grupos psicoeducativos que se han realizado en el marco del Programa ALFIL desde el año 1998 hasta el 2000, en total 13 grupos.

\subsection{Objetivos de la intervención}

La intervención psicoeducativa del Programa ALFIL tiene como objetivo general disminuir factores de riesgo y potenciar factores de protección en los hijos de alcohólicos y en sus familias. Este objetivo general se traduce en los siguientes propósitos específicos:

a) Promover en los jóvenes cambios en los conocimientos, las actitudes y las expectativas sobre el alcohol (y las otras drogas) para favorecer la toma de decisiones responsables y saludables con respecto a su consumo, y facilitar el entendimiento de los problemas que el alcohol ha oca- sionado en su familia. A nivel más operativo se pretende:

- Ofrecer información objetiva sobre el alcohol, sus efectos y los problemas derivados de su abuso, así como promover la crítica de los mitos culturales sobre el alcohol.

- Favorecer el entendimiento del alcoholismo como una enfermedad de desarrollo progresivo, que afecta a toda la familia, y que escapa del control de las personas que lo sufren, evitando actitudes estigmatizantes hacia los drogodependientes en general.

- Advertir, sin alarmar ni profetizar, que los hijos de alcohólicos pueden tener mayor riesgo de alcoholismo, en qué consiste ese riesgo y cómo se puede disminuir.

- Reforzar las actitudes de abstinencia de drogas en general y potenciar la capacidad de generar alternativas saludables para disfrutar del tiempo libre.

b) Facilitar el aprendizaje de estrategias de afrontamiento a los factores de riesgo individuales y ambientales que pueden llevar, entre otros problemas, a consumir drogas: dificultades emocionales y de autoestima, impulsividad, falta de habilidades sociales y de solución de problemas, situaciones de estrés ambiental, presión al consumo del grupo de amigos y de la publicidad, etc.

c) Utilizar el grupo como recurso educativo y terapéutico, procurando crear un clima de confianza y respeto mutuo que facilite:

- La identificación y el apoyo emocional entre los participantes, reduciendo la sensación de aislamiento generada por la necesidad de guardar "el secreto familiar".

- La discusión conjunta de opiniones propias y dudas respecto al alcohol, el alcoholismo y las otras drogodependencias.

- La demanda de ayuda a los demás ante los problemas personales.

- La práctica de habilidades sociales dentro de un grupo, en una situación controlada. 
- El aprendizaje de roles juveniles normativos a través de la identificación con modelos positivos dentro del grupo.

d) Facilitar una atención global a toda la familia con el fin de:

- Dotar a los padres de recursos educativos para establecer límites coherentes, mantener las normas y los valores familiares, fomentar la libertad y la responsabilidad progresivas en los hijos, entender la crisis adolescente y otros períodos de transición, mantener una actitud abierta y flexible con los hijos, potenciar las habilidades de comunicación, convivencia y resolución de conflictos.

- Disminuir los sentimientos de culpa sobre la enfermedad y los trastornos que ha podido ocasionar en la familia.

- Evitar las actitudes co-dependientes que facilitan el mantenimiento del hábito alcohólico.

- Favorecer la aceptación del alcohólico como un enfermo que, aunque no ha buscado conscientemente su enfermedad, debe responsabilizarse de su tratamiento y recuperación.

- Apoyar la reorganización familiar y el restablecimiento de los lazos afectivos

- Mostrar esperanza en el futuro.

En general, los contenidos del Programa ALFIL están centrados en la temática del alcohol; sin embargo, sus objetivos van más allá de la prevención del abuso de esta sustancia. En este programa hablar sobre el alcohol y el alcoholismo sirve, por un lado, para que los jóvenes y sus padres puedan entender cómo ha afectado a su vida familiar, y por otro, para educar en valores, actitudes y habilidades que pueden ayudar a prevenir, no solo problemas con el alcohol u otras drogas, sino cualquier tipo de psicopatología relacionada con dificultades emocionales y sociales que son frecuentes en las familias alcohólicas.

\subsection{Metodología}

La metodología del Programa ALFIL se describe en el manual que se ha utilizado como material de apoyo para la realización de grupos psicoeducativos para jóvenes con antecedentes familiares de alcoholismo. ${ }^{45}$ Las propuestas del manual, aunque suficientemente sistematizadas para permitir la evaluación de la experiencia, permiten hacer un uso flexible y acorde con las necesidades del grupo con el que se pretende trabajar. El manual también incluye cuestionarios para evaluar los cambios en los conocimientos, las actitudes y las expectativas sobre el alcohol, tras la participación en las sesiones; así como cuestionarios para recoger la valoración de la experiencia por parte de los padres y los hijos participantes.

Los grupos psicoeducativos del Programa ALFIL constan de 11 sesiones, 9 para los hijos y 2 para sus padres (Tabla 2). Los grupos de jóvenes están formados por entre 6 y 11 participantes, de ambos sexos, y de edades comprendidas entre los 8 y los 16 años divididos, siempre que es posible, en grupos de edad homogéneos. Las sesiones de grupo son guiadas por un educador-terapeuta (en ocasiones, se añade un co-terapeuta), tienen una duración aproximada de 90 minutos y se pueden realizar con una frecuencia de una o dos sesiones por semana. Si se dispone de personal suficiente, las sesiones para padres se suelen realizar paralelamente a la primera y la última sesión para hijos, aprovechando el final de la sesión para celebrar la despedida conjuntamente.

Las actividades que se realizan durante las sesiones siguen, fundamentalmente, una orientación cognitivo-conductual, aunque también hay lugar para el análisis de los sentimientos y las emociones más comunes en una familia con problemas de alcohol. Se procura mantener una dinámica de grupo participativa, fomentando la expresión de opiniones y experiencias personales. La información sobre el alcohol y otras drogas no se transmite unidireccionalmente, sino que se procura proporcionar a los jóvenes un espacio para que puedan compartir creencias y reflexionar sobre algunos conceptos, mitos y expectativas. También se les entrena en la toma de decisiones responsable después de discutir en grupo los pros y los contra del consumo de alcohol u otras drogas, así como en estrategias para hacer frente a las presiones internas y externas hacia ese consumo. 


\begin{tabular}{|c|c|c|}
\hline \multicolumn{3}{|c|}{ SESIONES PARA PADRES } \\
\hline Sesión & Título & Contenidos básicos \\
\hline 1 & $\begin{array}{l}\text { Educar a los hijos para prevenir: } \\
\text { una tarea difícil }\end{array}$ & $\begin{array}{l}\text { Características de los adolescentes } \\
\text { Pautas educativas saludables (normas, valores, libertad y respon- } \\
\text { sabilidad progresivas, comunicación, etc.) }\end{array}$ \\
\hline 2 & $\begin{array}{l}\text { ¿Cómo hablar con los hijos del } \\
\text { alcohol y otras drogas? }\end{array}$ & $\begin{array}{l}\text { La conveniencia de hablar de la propia experiencia con el alcohol. } \\
\text { Manejar la vergüenza y la culpa. Pautas de actuación ante el con- } \\
\text { sumo de drogas de los hijos }\end{array}$ \\
\hline \multicolumn{3}{|c|}{ SESIONES PARA HIJOS } \\
\hline Sesión & Título & Contenidos básicos \\
\hline 1 & $\begin{array}{l}\text { Opiniones sobre el alcohol } \\
\text { y sus efectos }\end{array}$ & Formas de uso, abuso y dependencia. \\
\hline 2 & $\begin{array}{l}\text { Información objetiva sobre el } \\
\text { alcohol (los mitos y la realidad) }\end{array}$ & $\begin{array}{l}\text { Crítica de los mitos culturales. } \\
\text { Efectos objetivos del alcohol: agradables y desagradables, orgáni- } \\
\text { cos y psicológicos, a corto y largo plazo. }\end{array}$ \\
\hline \multirow[t]{2}{*}{3} & $\begin{array}{l}\text { El alcoholismo, una enfermedad } \\
\text { con tratamiento }\end{array}$ & $\begin{array}{l}\text { La progresión del uso al abuso y la dependencia. } \\
\text { El alcoholismo como enfermedad } \\
\text { Tratamiento y rehabilitación. El mito de la "no-curación" }\end{array}$ \\
\hline & $\begin{array}{l}\text { ¿Por qué empiezan a beber } \\
\text { algunos jóvenes? }\end{array}$ & $\begin{array}{l}\text { Factores de riesgo y factores de protección. } \\
\text { Entrenamiento en técnicas de afrontamiento cognitivo-conduc- } \\
\text { tuales y emocionales: }\end{array}$ \\
\hline 4 & $\begin{array}{l}\text { - La presión del grupo de amigos } \\
\text { y de la publicidad }\end{array}$ & $\begin{array}{l}\text { - Toma de decisiones } \\
\text { - Analizar y afrontar la presión del grupo y de la publicidad }\end{array}$ \\
\hline 5 & - El malestar emocional & $\begin{array}{l}\text { - Afrontar el estrés, aumentar la autoestima, expresar senti- } \\
\text { mientos, resolver problemas, planificar el futuro, etc. }\end{array}$ \\
\hline 6 & $\begin{array}{l}\text { - La facilitación de las relaciones } \\
\text { sociales }\end{array}$ & $\begin{array}{l}\text { - Habilidades sociales: comunicación asertiva, ligar y hacer ami- } \\
\text { gos, manejo de conflictos, etc. }\end{array}$ \\
\hline 7 & $\begin{array}{l}\text { El alcoholismo, una enfermedad } \\
\text { "familiar": factores de riesgo en } \\
\text { la familia y repercusiones en la } \\
\text { convivencia. }\end{array}$ & $\begin{array}{l}\text { El mito de "la herencia del alcoholismo" } \\
\text { Un factor de riesgo genético: "aguantar bien el alcohol" } \\
\text { El secreto familiar: "un elefante en el salón" } \\
\text { Malestar emocional ante situaciones típicas en el hogar alcohóli- } \\
\text { co (mentiras, olvidos, agresividad, etc.) } \\
\text { Factores de protección en la familia }\end{array}$ \\
\hline 8 & $\begin{array}{l}\text { Los síntomas iniciales de } \\
\text { problemas con el alcohol en } \\
\text { los jóvenes. }\end{array}$ & $\begin{array}{l}\text { Los primeros síntomas: "yo aguanto mucho...," "necesito el pun- } \\
\text { tillo para pasarlo bien y evadirme..." } \\
\text { Los límites entre el uso y el abuso } \\
\text { ¿Cómo ayudar a alguien con problemas con el alcohol? }\end{array}$ \\
\hline 9 & Despedida & $\begin{array}{l}\text { Últimas dudas y comentarios. Resumen de ideas clave (póster). } \\
\text { Evaluación de las sesiones y celebración padres-hijos }\end{array}$ \\
\hline
\end{tabular}

Los datos para evaluar las sesiones educativas que se presentan en este trabajo fueron obtenidos a través de tres procedimientos: a) Cuestionarios pre-post sesiones sobre conocimientos, actitudes y expectativas sobre el alcohol. 
b) Cuestionarios de valoración de las sesiones para padres y para hijos

c) Talleres de valoración de la experiencia por parte de los profesionales implicados ("focus group")

La asignación de los sujetos a los grupos de intervención se realizó de forma quasi-aleatoria. Durante la sesión de evaluación previa de los participantes en el estudio descriptivo del programa ${ }^{70,71}$ se informaba a los padres acerca de la posibilidad de participar en una serie de sesiones educativo-preventivas, para padres e hijos por separado. También les indicábamos que el programa de sesiones educativas se iba repitiendo a lo largo del tiempo, de modo que si no les avisábamos para el primero que se realizase, podíamos avisarles más adelante. Cuando las familias mostraban resistencias a participar, se utilizaban estrategias motivacionales para promover cambios de actitud que facilitasen su aceptación de la intervención. ${ }^{45}$ De esta manera, se formaba una "lista de espera" de familias candidatas a la intervención, a las que se iba llamando por teléfono para formar los sucesivos grupos, aleatoriamente. Algunas de las familias de esta lista aún no han podido participar en grupos psicoeducativos, debido a problemas de incompatibilidad horaria con el trabajo de los padres, con actividades extraescolares de los hijos, negativa de los hijos a participar, etc. Una selección de estas familias, emparejadas con el grupo experimental, constituirán el grupo control con el que se pretende comparar el grupo de intervención transcurridos 5 años desde la realización de las intervenciones.

\subsection{Resultados de la evaluación y discu- sión}

Aunque el trabajo que se expone aquí no es, estrictamente, un artículo de investigación, puede resultar de interés comentar algunos de los resultados de la evaluación del proceso y la eficacia de los grupos psicoeducativos para hijos de alcohólicos y sus padres que se han realizado en el marco del Progra- ma ALFIL desde marzo del año 1998 hasta marzo del año 2000.

Como era de esperar, esta evaluación ha presentado las dificultades metodológicas propias de los programas preventivos experimentales dirigidos a grupos de alto riesgo. ${ }^{72}$ El análisis de algunas de estas dificultades se presenta a continuación, por su utilidad potencial para optimizar la metodología y el procedimiento de evaluación de este tipo de programas en el futuro.

Cumplimiento de la organización y planificación de actividades

Tras la realización de tres grupos piloto en Barcelona, Zaragoza y Alcoy, utilizando un manual preliminar y una reunión de intercambio de materiales y de experiencias previas, se elaboró el manual definitivo del programa y se realizó un taller de formación para nuevos profesionales interesados, con objeto de extender las sesiones psicoeducativas al resto de los centros participantes en el proyecto (véase la Tabla 3 ).

Entre los meses de marzo de 1998 y marzo del 2000 se realizaron los 13 grupos psicoeducativos que se habían previsto en los 7 centros participantes hasta ese momento. Todos ellos se iniciaron con ligero retraso respecto a la planificación temporal inicial, debido a dificultades para obtener el material necesario idéntico para todos los centros, problemas para acceder o motivar a la población diana a participar en esta experiencia piloto, dificultades para contactar con las familias por teléfono en horario de trabajo, coincidencia con épocas de exámenes o actividades extraescolares de los niños, vacaciones escolares, etc.

\section{Descripción de los grupos realizados}

En total han participado en los grupos psicoeducativos 97 hijos de alcohólicos (49.2\% de sexo masculino) y 146 padres $(38.5 \%$ de sexo masculino) (Tabla 3).

En un principio, las sesiones fueron diseñadas para grupos de adolescentes de 12 a 16 
Tabla 3. Descripción de los grupos realizados en el Programa ALFIL desde marzo de 1998 hasta marzo del 2000

\begin{tabular}{|c|c|c|c|c|c|}
\hline \multirow[t]{2}{*}{ Centro } & \multirow[t]{2}{*}{ Grupo } & \multirow[t]{2}{*}{ Fechas } & \multicolumn{2}{|c|}{ Hijos } & \multirow{2}{*}{$\begin{array}{l}\text { Padres } \\
\text { Partic }^{* 2}\end{array}$} \\
\hline & & & Partic ${ }^{* 1}$ & Edad (años) & \\
\hline Alcoi (Alicante) & 1 & May 98 & 10 & $11-16$ & 12 \\
\hline Alcoi (Alicante) & 2 & Dic 98 & 8 & $9-16$ & 13 \\
\hline Alcoi (Alicante) & 3 & Mar 99 & 9 & $11-15$ & 10 \\
\hline Barcelona & 4 & May 98 & 7 & $12-16$ & 8 \\
\hline Barcelona & 5 & Feb 99 & 8 & $11-17$ & 10 \\
\hline Barcelona & 6 & Mar 00 & 8 & $11-16$ & 9 \\
\hline Córdoba & 7 & Mar 99 & 6 & $12-15$ & 6 \\
\hline Madrid & 8 & Ene 99 & 11 & $10-16$ & 14 \\
\hline Santiago (Coruña) & 9 & Ene 99 & - & - & - \\
\hline Torrent (Valencia) & 10 & Mar 98 & 8 & $10-15$ & 10 \\
\hline Torrent (Valencia) & 11 & Mar 99 & 8 & $9-12$ & 11 \\
\hline Zaragoza & 12 & Mar 98 & 7 & 8-16 & 9 \\
\hline Zaragoza & 13 & Feb 99 & 7 & 8-16 & 8 \\
\hline \multicolumn{3}{|c|}{ Total participantes } & $\begin{array}{c}97 \\
\text { (49.2\% masc.) }\end{array}$ & $\begin{array}{c}\text { 8-17 } \\
(13.5 \pm 2.17) \\
\end{array}$ & $\begin{array}{c}146 \\
\text { (38.5\% masc.) }\end{array}$ \\
\hline \multicolumn{3}{|c|}{ Total cuestionarios entregados ${ }^{* 3}$} & 80 & & 84 \\
\hline
\end{tabular}

*1: Número de jóvenes que asistieron a un mínimo de 4 sesiones

*2: Número de padres que asistieron, como mínimo, a una sesión

*3: Número de cuestionarios de valoración de las sesiones entregados en la última de las sesiones del programa.

años. Sin embargo, tras las primeras experiencias, se comprobó que si se intervenía más tempranamente era posible captar a los jóvenes con tendencias disociales que, llegados a la adolescencia, se negaban a acudir a las sesiones; de manera que se adaptaron algunos contenidos para niños más pequeños. Por otro lado, en general, los padres que tenían dos o más hijos manifestaban su deseo de que éstos acudieran juntos a las sesiones, a pesar de las diferencias de edad. Finalmente se permitió la participación de jóvenes con edades comprendidas entre los 8 y los 17 años, intentando agruparlos por edades homogéneas siempre que era posible (Tabla 3).

El porcentaje medio de asistencia de los jóvenes a las sesiones fue del 79.2\% (un promedio de $7.13 \pm 1.75$ sesiones cada niño), según los datos recogidos en los 9 grupos en los que se anotaron sistemáticamente los asistentes a cada sesión. En general, los niños avisaron de su ausencia en días concretos por causas justificadas.

En 11 de los 13 grupos realizados, las sesiones se desarrollaron con normalidad, según las directrices del manual. En algunos casos se introdujeron pequeñas modificaciones en las actividades propuestas o en los materiales, con el fin de adaptarse a las características de cada grupo concreto y a la experiencia del profesional, aunque siempre manteniendo los objetivos y los contenidos básicos de cada sesión. En los dos grupos restantes los profesionales encargados de realizar las sesiones revelaron algunas dificultades para realizar la totalidad de éstas. Estas dificultades fueron atribuidas a la dispersión de la población diana (zonas rurales), la falta de medios de transporte público, la falta de personal de apoyo en los centros y a la falta 
de experiencia en el uso de las técnicas motivacionales, entre otras razones.

Tras realizar las primeras experiencias piloto con grupos psicoeducativos para hijos de alcohólicos, en diferentes centros se han realizado nuevas actividades de prevención selectiva adaptadas a las características socioculturales de la población que atienden, a las particularidades de los jóvenes que componen cada grupo concreto, así como a la organización de la red de asistencia sociosanitaria de cada comunidad. Las modificaciones realizadas han surgido de un análisis conjunto de las dificultades y de la propuesta de diferentes soluciones a los problemas detectados en las reuniones que los profesionales que participan en el Programa ALFIL han mantenido a lo largo de estos años.

Análisis de dificultades y soluciones adoptadas

Uno de los principales obstáculos que encontramos para realizar grupos psicoeducativos con hijos de alcohólicos y con sus padres fue el manejo de las resistencias que suelen mostrar estas familias a participar en este tipo de actividades, especialmente las familias que presentan mayor riesgo. Muchos alcohólicos se sienten culpables del sufrimiento que han ocasionado en sus familiares y esto hace que no sean capaces de reconocer la presencia de problemas en su familia, otros creen que sus hijos no han captado nada anormal en su comportamiento, o que aún son demasiado pequeños para poder hablar de estos temas. Por su parte, algunos hijos muestran resentimiento hacia sus padres y se niegan a acudir a las sesiones, alegando que no son ellos los que tienen que resolver los problemas de su casa, sino sus padres. Todos ellos suelen utilizar, como mecanismo de defensa, la negación de los problemas familiares, y muestran una gran dificultad para destapar el cúmulo de sentimientos dolorosos y contradictorios que ha ocasionado la enfermedad en su familia. En el manual del Programa ALFIL se ofrecen algunas directrices sobre cómo abordar las resistencias típicas de estas familias. ${ }^{45}$ Duran- te estos años también se ha procurado facilitar el entrenamiento en técnicas motivacionales de los profesionales interesados en realizar este tipo de intervenciones, a través de talleres y seminarios especializados. El siguiente paso para conseguir avanzar en este campo será la formación de los agentes sociales que podrían facilitar la detección de los jóvenes procedentes de familias con problemas de alcohol que no reciben atención específica, con el fin de facilitar su acceso a programas educativos y/o terapéuticos, incluso en el caso de que el progenitor alcohólico no esté recibiendo un tratamiento específico para su adicción.

Otra de las dificultades encontradas fue la falta de materiales adaptados a nuestro entorno socio-cultural para abordar con los hijos de alcohólicos temas clave como la codependencia, la culpa, la vergüenza, el estigma de la enfermedad alcohólica, los roles desadaptativos en los hijos de alcohólicos, los conflictos típicos en la familia alcohólica, los factores de riesgo genéticos en el alcoholismo, etc. Por ello, hemos iniciado la elaboración de materiales preventivos específicos para las sesiones, así como de materiales informativos y de autoayuda apropiados para estas familias, como el folleto "¿Alcohol en la familia?"." ${ }^{3}$

Algunos de los educadores manifestaron también dificultades para hacer participar activamente a los jóvenes en la dinámica de las sesiones de grupo, especialmente durante las primeras sesiones, si es que no se conocían previamente. Para tratar de solucionar este problema típico en los grupos con hijos de alcohólicos, ${ }^{65}$ además de la necesaria preparación de los educadores en técnicas de dinamización de grupos de jóvenes, en algunos centros resultó de gran ayuda guiar los grupos en la modalidad de coterapia. Esta modalidad, además de facilitar la supervisión de las sesiones, permite colaborar en la preparación de las actividades, así como realizar escenificaciones y bromas entre los educadores-terapeutas para hacer más amenas y distendidas las sesiones, especialmente cuando 
se tratan temas que pueden crear malestar emocional entre los participantes.

Otro aspecto que, en principio, pareció problemático fue la dificultad para formar grupos de jóvenes de edades similares. Sin embargo, en la mayoría de ocasiones, la presencia en los grupos de jóvenes de diferentes edades resultó positiva en el sentido de que los mayores servían de modelo respetable para los pequeños, y se establecían relaciones de enseñanza-aprendizaje mutuas muy satisfactorias. Para los más jóvenes participar en una experiencia de este tipo con jóvenes de mayor edad resultaba agradable, pues les hacía sentir mayores. Los jóvenes de mayor edad, especialmente las chicas, se mostraban dispuestos a ayudar y enseñar algunas cosas a los más pequeños, aunque en general mostraban preferencia por participar en grupos de discusión a un nivel más profundo con jóvenes de su misma edad. Una manera de conseguir grupos más homogéneos en edad sería realizar las sesiones en lugares centralizados que permitieran reunir a la población diana de diversos centros de tratamiento, asociaciones de alcohólicos rehabilitados, etc. Otra forma de homogeneizar los grupos sería realizarlos dentro del marco escolar, a través del procedimiento de selección de jóvenes de riesgo por parte de los profesores, ${ }^{59,62}$ del procedimiento de autoselección de jóvenes preocupados por el consumo de alcohol de sus familiares o amigos, o bien a través de un cuestionario breve de screening..$^{56,57}$

Otro problema frecuente en los grupos de alto riesgo realizados fuera del entorno escolar es la asistencia irregular a las sesiones por parte de algunos jóvenes, a pesar de hacerles énfasis en la conveniencia de acudir a todas ellas. En general, en los grupos realizados, las ausencias fueron debidas a causas médicas o actividades extraescolares. En algunas ocasiones se debieron a olvidos de los niños o de los padres. Probablemente también influyeron las resistencias inconscientes a abordar algunos temas conflictivos o que generan cierto malestar emocional. En definitiva, en cualquiera de los casos, en los grupos educa- tivos que cuentan con un número concreto de sesiones, en los que todos los niños deben recibir la misma información y realizar las mismas actividades, es esencial que los niños acudan al mayor número posible de sesiones. Por lo tanto, es necesario utilizar técnicas de seguimiento y motivación a través del teléfono (por ejemplo, cuando se sospecha que un niño no va a acudir), el uso contingente de refuerzos como meriendas, pequeños obsequios, uso libre de internet o juegos de ordenador tras la sesión, etc. Para cubrir la ausencia de algunos niños a determinadas sesiones, en ocasiones es necesario reorganizarlas sobre la marcha, y suele ser muy útil realizar resúmenes y recapitulaciones periódicas de lo acontecido en las sesiones anteriores antes de comenzar una nueva sesión.

En relación con el tema anterior, resultó especialmente difícil motivar a participar en los grupos a los adolescentes que presentaban características disociales, que son precisamente los que presentan mayor riesgo de consumo de drogas y delincuencia. De hecho, la mayoría de estos jóvenes ya habían iniciado el consumo de sustancias adictivas y podían pensar que en las sesiones de grupo se les iba a recriminar por ello. En los 9 grupos en los que se anotaron sistemáticamente los jóvenes que asistieron a cada sesión, 7 jóvenes que presentaban características disociales no llegaron a acudir ni siquiera a la primera sesión, a pesar del interés y la insistencia de sus padres. Por otro lado, los jóvenes con estas características que acudían a los grupos, solían hacerlo muy irregularmente y requerían excesiva atención por parte del guía. En general, no se adaptaban fácilmente a la disciplina que requiere el trabajo en grupo, mostrando numerosas conductas disruptivas. En el manual del programa se ofrecen algunas directrices prácticas para tratar este tipo de problemas. ${ }^{45}$ En algunos grupos, para captar este tipo de casos de "riesgo extremo" se optó por ampliar la edad de intervención hacia los más jóvenes (8 a 12 años), ya que a estas edades aún no han iniciado el consumo de sustancias, y son más 
influenciables por las directrices paternas. Otra solución posible para facilitar la inclusión de estos casos en los grupos educativos, sería realizar inicialmente un abordaje individual con el fin de prepararlos para su posterior participación en un grupo.

Para terminar, en la Figura 2 se puede apreciar que al $41.7 \%$ de los padres la duración de las sesiones del programa dedicadas a ellos les pareció corta, y al 10.7 \% muy corta. En general, los padres mostraron su deseo de participar en nuevas sesiones educativas, alegando que en dos sesiones no tenían tiempo suficiente para hablar de sus preocupaciones y dificultades en relación con la educación de sus hijos en temas de drogas. Por ello, se decidió iniciar la experiencia de "escuela de padres " dirigida específicamente a pacientes con problemas de alcohol y a sus parejas. Hasta el momento, se han realizado experiencias piloto de este tipo de grupos en dos de los centros, con resultados muy positivos.

\section{Figura 2. Valoración de la duración de las sesiones del programa, según padres e hijos.}

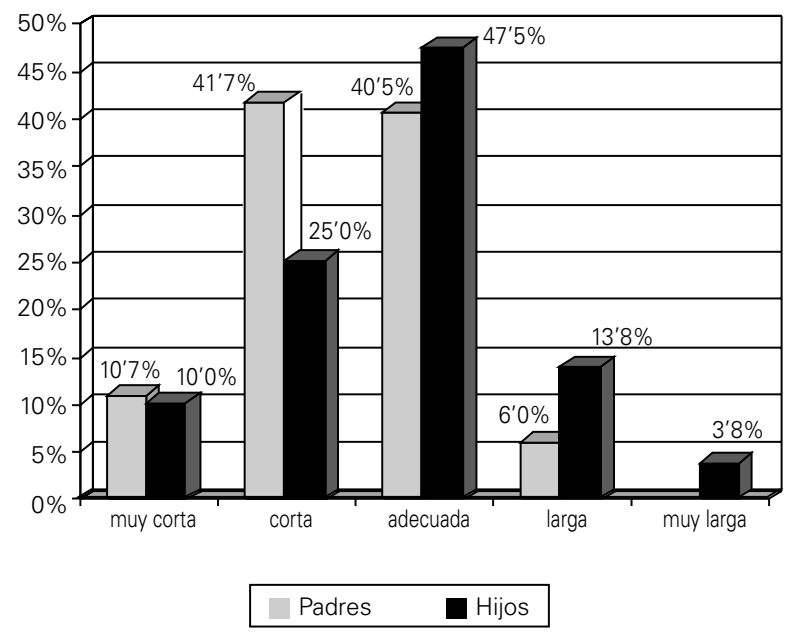

Interés social y difusión posterior del programa

A medida que se ha ido conociendo la existencia del Programa ALFIL, a través de la difusión de trípticos informativos para pacientes y profesionales, la participación en congresos nacionales e internacionales, y la impartición de charlas y conferencias, entre otras actividades, ha ido aumentado la demanda de participación por parte de los afectados. También ha aumentado paralelamente la petición de manuales del programa y materiales específicos, los talleres de formación e intercambio de experiencias, y las demandas de asesoramiento sobre casos particulares por parte de profesionales interesados en realizar actividades preventivas con hijos de alcohólicos y con sus padres. Uno de los colectivos más interesados en el programa ha sido el de las asociaciones de alcohólicos rehabilitados, centradas durante años en la labor de información y sensibilización de la población para lograr la reincorporación social del enfermo alcohólico y de sus familiares.

En relación con la difusión del programa, también se debe hacer constar que en los últimos años se han incorporado al Programa 
ALFIL nuevos colaboradores que, junto con los pioneros, están realizando nuevos grupos con padres e hijos, así como actividades de divulgación, sensibilización y formación de profesionales para extender este tipo de experiencias educativas para hijos de alcohólicos en diferentes ámbitos de atención social, educativa y sanitaria de las diferentes comunidades españolas ("5 y más, servicios socio-culturales" de Salamanca, "Fundación AEPA" de la Comunidad Valenciana, "Instituto Bitácora" de Sevilla, etc.). Igualmente, se han establecido contactos con profesionales europeos e iberoamericanos con el fin de realizar intercambios de experiencias y materiales, así como proyectos multicéntricos de alcance internacional.
Valoración subjetiva de los participantes

El análisis descriptivo de los datos de los cuestionarios de evaluación completados por los padres y los hijos participantes tras la realización de las sesiones, reveló que participar en esta experiencia había resultado bastante o muy satisfactorio para el $85.7 \%$ de los padres y para el $82.6 \%$ de los hijos que entregaron el cuestionario de valoración en la última sesión (un total de 80 hijos y 84 padres) (Figura 3). Otros aspectos valorados, como la cantidad y la claridad de la información, o la utilidad de las actividades propuestas, obtuvieron igualmente resultados muy positivos.

\section{Figura 3. Satisfacción general de padres e hijos con su participación en las sesiones.}

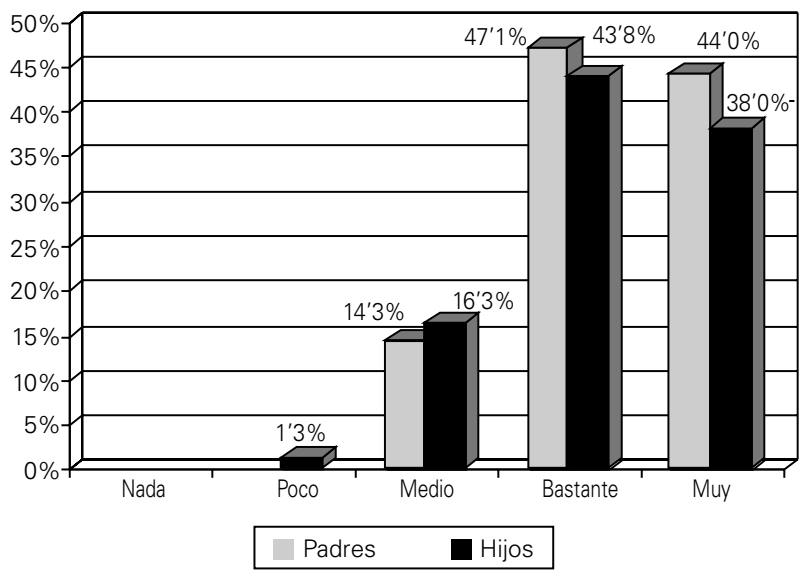

\section{Eficacia de las sesiones}

Por el momento, se dispone de algunos datos cuantitativos para valorar la eficacia inmediata de las sesiones psicoeducativas en el cumplimiento de algunos de los objetivos propuestos, como el cambio en las cogniciones sobre el alcohol y otras drogas, y los cambios en la dinámica familiar (grado de comunicación y conflictos).

El análisis de la varianza de medidas repetidas pre-post sesiones de los indicadores globales de conocimientos, actitudes y expecta- tivas sobre el alcohol, calculados a partir de las respuestas de los sujetos a tres cuestionarios de 36, 37 y 30 ítems, respectivamente, ${ }^{45}$ reveló diferencias significativas en el índice global de conocimientos $(p<0.001$ ) (Figura 4 ), aunque no en la actitud general hacia el consumo, ni en las expectativas sobre el alcohol, de modo similar a lo que ocurre en otros estudios sobre intervenciones preventivas en hijos de alcohólicos. ${ }^{56}$

Según la literatura revisada, en general, los hijos de alcohólicos en edad escolar mues- 
tran actitudes y expectativas sobre el alcohol bastante negativas. Sin embargo, esto no les protege de desarrollar problemas relacionados con el consumo de esa sustancia en el futuro, ya que a medida que se acerca la adolescencia sus actitudes y expectativas cambian hasta alcanzar posiciones más favorables hacia el consumo, quizás debido a que en los primeros contactos con la sustancia suelen tener una respuesta más satisfactoria que los jóvenes de la población general. ${ }^{74}$

Por otro lado, en este estudio, el hecho de evaluar a los hijos de alcohólicos en el centro al que acuden sus padres también ha podido influir en que simularan actitudes y expectativas bastante negativas incluso antes de iniciar las sesiones, lo que también explicaría la ausencia de diferencias pre-post. Por otro lado, en el programa se pretendía ofrecer una visión realista de los efectos del alcohol, aceptando que algunos de estos efectos son percibidos de forma positiva o satisfactoria por los consumidores, al menos al principio; y que éste es precisamente uno de los factores que pueden determinar el consumo habitual y el abuso de la sustancia.

Todas las razones anteriores permitirían explicar la ausencia de cambios significativos tras las sesiones en las actitudes y expectati- vas sobre el alcohol en los jóvenes estudiados. Para poder profundizar en el conocimiento de los factores que pueden llevar a estos jóvenes a consumir alcohol, a pesar de sus actitudes negativas iniciales, se deberá realizar un seguimiento de los sujetos para obtener nuevos datos a lo largo de la adolescencia y post-adolescencia sobre sus intenciones de consumir alcohol, su consumo real, los efectos que les produce y las razones que les llevan consumir ésta u otras sustancias, con el fin de relacionarlos con la presencia de algunos marcadores de riesgo obtenidos en la evaluación previa.

En relación con la dinámica de las familias que participaron en los grupos, también se detectaron algunos cambios que se han podido cuantificar. Por un lado, el $55.7 \%$ de los padres y el $30 \%$ de los hijos informaron de mayor diálogo en casa tras la participación en las sesiones. Por otro lado, el $26.2 \%$ de los padres y el $23.8 \%$ de los hijos informaron de menos enfrentamientos tras la participación en las sesiones. Y por último, el $52.4 \%$ de los padres y el $28.8 \%$ de los hijos informaron de la puesta en práctica de las recomendaciones y técnicas sugeridas en las sesiones (aunque el $10.7 \%$ y el $5 \%$ respectivamente indicaron que no habían sido efectivas).

Figura 4. Cambio pre-post sesiones en el índice de conocimientos sobre el alcohol. Este índice se obtiene sumando el total de ítems con respuesta correcta y restando la mitad de los ítems en blanco, de un total de 36 ítems $(p \leq 0.001)$

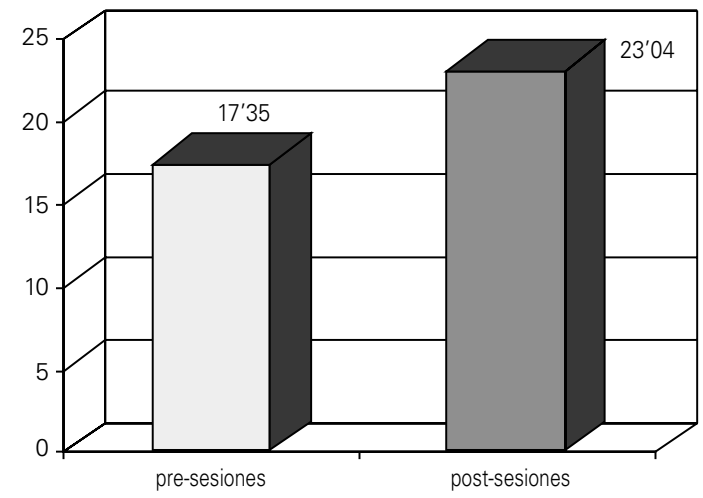


A nivel cualitativo, en algunas familias se observaron cambios en las actitudes de las parejas de los enfermos alcohólicos al tomar un papel más activo a la hora de buscar atención especializada para sus hijos, a pesar de la oposición inicial del progenitor alcohólico. Aunque no disponemos de datos exactos, algunos niños y familiares fueron derivados a centros especializados por considerar que requerían algún tipo de terapia adicional, y al menos 4 progenitores alcohólicos reiniciaron su tratamiento durante la participación de su familia en los grupos educativos, tras haberlo abandonado debido a una recaída. En próximos estudios de evaluación de los programas preventivos para hijos de alcohólicos y sus familiares, se debería cuantificar este tipo de datos con el fin de captar mejor posibles cambios en la dinámica familiar, que pueden constituir factores moderadores del riesgo o bien factores de protección para los hijos.

Por último, para la valoración de la eficacia a medio-largo plazo de las sesiones será preciso el seguimiento de los participantes y su comparación con un grupo control equivalente de hijos de alcohólicos que no haya participado en los grupos educativos, con el fin de comprobar si se mantienen los cambios en los conocimientos sobre el alcohol, presentan mejor ambiente familiar, menos consumo de alcohol u otras drogas, menos psicopatología y problemas psicosociales, mejor rendimiento cognitivo y escolar y mejor evolución del alcoholismo paterno, entre otros indicadores de eficacia.

\section{CONCLUSIONES}

Después de algo más de cuatro años de investigación sobre intervenciones preventivas para hijos de alcohólicos realizadas en varias comunidades de nuestro entorno sociocultural, es posible empezar a responder algunas de las preguntas que se plantearon al iniciar la experiencia del Programa ALFIL. ¿Necesitan los hijos de alcohólicos una intervención con características específicas?.
¿Debe dirigirse esa intervención a todos los hijos de alcohólicos? ¿Qué hijos de alcohólicos necesitan una intervención más intensa?. ¿Cuándo y cómo debemos intervenir? ¿Cuáles son los lugares más apropiados? ¿Qué agentes sociales deberían estar implicados?

Por su especial vulnerabilidad para sufrir consecuencias negativas relacionadas con el consumo de alcohol y otras drogas, los hijos de alcohólicos son una población diana para la prevención selectiva. Tanto en el nuestro como en otros países, constituyen un grupo suficientemente amplio para justificar la realización de esfuerzos preventivos específicos, que pueden ser incluso más rentables que los dirigidos a la población general o a otros grupos de riesgo menos numerosos. La intervención a través de grupos psicoeducativos podría ser suficiente en la mayoría de los casos para disminuir de una forma significativa los factores de riesgo y aumentar los de protección. Sin embargo, los casos más vulnerables requerirían ser identificados lo más precozmente posible para realizar una intervención más intensa e individualizada.

La metodología óptima para intervenir preventivamente con los hijos de alcohólicos requiere un plan integral de intervención que incluya diferentes acciones: detección precoz, motivación al cambio en la familia, educación sobre temas relacionados con el alcoholismo familiar $y$, en caso necesario, actividades terapéuticas más intensivas para toda la familia. Cada uno de los agentes sociales en contacto con esta población tendría un papel específico en este plan de intervención. Los maestros, los agentes del orden y el personal socio-sanitario de atención primaria serían los encargados de la detección precoz. Los profesionales especializados en la prevención y el tratamiento del alcoholismo y en la atención a los familiares podrían realizar la intervención psicoeducativa. Por último, los psiquiatras y psicólogos clínicos serían los más adecuados para realizar intervenciones terapéuticas más intensas e individualizadas, en los casos de mayor riesgo, en coordinación con los especialistas en el tratamiento del alcoholismo. Para conseguir que este plan 
integral funcione sería imprescindible realizar tareas de sensibilización y motivación de todos los agentes preventivos implicados, con un especial énfasis en la coordinación entre los diferentes servicios socio-sanitarios encargados de cada una de las acciones propuestas.

La evaluación de la experiencia del Programa ALFIL hasta el momento ha demostrado que los grupos psicoeducativos para hijos de alcohólicos son, en general, bien aceptados, y tienen resultados positivos. El análisis de las dificultades que ha supuesto la realización de este tipo de intervenciones en algunos centros de atención al alcoholismo ha permitido introducir progresivamente innovaciones y mejoras que pueden facilitar no sólo la realización de grupos para hijos de alcohólicos, sino también la adaptación de este tipo de programas a otros jóvenes de alto riesgo.

En los próximos años, la investigación sobre intervenciones preventivas en hijos de alcohólicos deberá enfocarse hacia las siguientes áreas:

a) Diseño de instrumentos y técnicas para la detección precoz y para la evaluación sistemática de factores de riesgo y protección.

b) Avances en estrategias motivacionales específicas para atraer a las familias y retenerlas en los programas.

c) Diseño y adaptación de materiales preventivos y de autoayuda específicos.

d) Evaluación continuada y diseminación de grupos psicoeducativos para hijos de alcohólicos y para sus padres.

Sin embargo, si se pretende ir un paso más allá y aplicar los resultados de la investigación a la atención socio-sanitaria del alcoholismo, será precisa la extensión y la adaptación flexible de este tipo de programas a las diferentes características de nuestro entorno sociocultural, lo que requerirá una mayor experimentación e intercambio de experiencias, además de un esfuerzo específico de formación de profesionales en esta área. Para ello será necesario un apoyo institucional que permita definir claramente cuáles serán las responsabilidades de los diferentes agentes sociales involucrados y facilitar su adecuada formación y coordinación para evitar multiplicar esfuerzos.

El Programa ALFIL, que se inició como un proyecto de investigación para evaluar la posible utilidad de los grupos psicoeducativos para hijos de alcohólicos en nuestro país, ha constituido, tras la experiencia piloto, una respuesta a una necesidad social evidente, si tenemos en cuenta la demanda y el interés suscitado por el programa. Por ello, prosiguen las actividades de investigación, diseminación y formación de profesionales que permitirán continuar avanzando hacia mejores prácticas preventivas para los hijos de alcohólicos y otros grupos de alto riesgo en nuestro entorno socio-cultural.

\section{AGRADECIMIENTOS}

El trabajo de investigación a que se refiere este capítulo ha sido subvencionado a Socidrogalcohol por la Delegación del Gobierno para el Plan Nacional sobre Drogas (19972001).

Agradecemos sus aportaciones a todos los profesionales de Socidrogalcohol y a todos los niños y familias que han participado en el Programa ALFIL.

\section{BIBLIOGRAFÍA}

(1) McNamee, J.E. y Offord, D.R. Children of alcoholics. En Canadian Task Force on the Periodic Health Examination. Canadian Guide to Clinical Preventive Health Care. Otawa: Health Canada. 1994 (pp. 470-485)

(2) Ackerman, R. J. Children of alcoholics. A guide for parents, educators and therapists. 2nd edition. A Learning Publication. New York: Firesid, Simon and Schuster. 1987.

(3) Alarcón, C., Ferrer, X., y Sierra, E. Problemática de los hijos de alcohólicos. VIII Jornadas 
Nacionales de Socidrogalcohol. Gandía (Valencia). 1980.

(4) Steinhausen, H.C. Children of alcoholic parents. Acta Paediatrica, 1993, 82, 208-209.

(5) Ellis, D.A. Zucker, R.A., y Fitzgerald, H.E. The role of family influences in development and risk. Alcohol, Health and Research World, 1997, 21 (3), 218-226.

(6) Díaz, R., Polo, M.D., Escera, C., Sánchez-Turet, M., y Grau, C. Marcadores neurogenéticos de riesgo para el alcoholismo. En M. SánchezTuret (Ed.), Enfermedades y problemas relacionados con el alcohol. Barcelona: Ediciones ESPAXS. 1999 (pp. 63-111).

(7) McGue, M. A behavioral-genetic perspective on children of alcoholics. Alcohol, Health and Research World, 1997, 21 (3), 210-217.

(8) Schuckit, M.A. A clinical model of genetic influences in alcohol dependence. Journal of Studies on Alcohol, 1994, 55, 5-17.

(9) Abel, E.L. Paternal exposure to alcohol. En T.B. Sonderegger (Ed), Perinatal substance abuse: Research findings and clinical implications. Baltimore: John Hopkins University Press. 1992 (pp 132-160).

(10) Durcan, M.J., y Goldman, D. Genomic imprinting: implications for behavioral genetics. Behavioral Genetics, 1993, 23, 137-143.

(11) Díaz, R. Las repercusiones del alcoholismo paterno en la descendencia: Avances hacia la prevención en una población de riesgo. Aula Médica Psiquiátrica, 1999, 1, 51-61.

(12) Gual A., y Díaz, R. Alcoholism Prevention in children of alcoholics. Ponencia en el Symposium "Alcohol dependence: Epidemiological and social problems". VII Congress of the European Society for Biomedical Research on Alcoholism (ESBRA). Barcelona. Junio 1999. (Resumen publicado en Alcohol and Alcoholism, 1999, 34 (3), 440.

(13) Sher, K.J., Gershuny, B.S., Peterson, L., y Raskin, G. The role of childhood stressors in the intergenerational transmission of alcohol use disorders. Journal of Studies on Alcohol, 1997, 58, 414-427.

(14) Adger, H. The role of primary care physicians. http:www.health.org/nacoa. 1997

(15) Weinberg, N.Z. Cognitive and behavioral deficits associated with parental alcohol use. Journal of the American Academy of Child and Adolescent Psychiatry, 1997, 36 (9), 11771186.

(16) Clark, D.B., Moss, H.B., Kirisci, L., Mezzich, A.C., Miles, R., y Ott, P. Psychopathology in preadolescent sons of fathers with substance use disorders. Journal of the American Academy of Child and Adolescent Psychiatry, 1997, 36 (4), 495-502.

(17) Hawkins, C.A. Disruption of family rituals as a mediator of the relationship between parental drinking and adult adjustment in offspring. Addictive Behaviours, 1997, 22 (2), 219-231.

(18) Hill, E.M., Thomson Ross, L., Mudd, S.A., Y Blow, F.C. Adulthood functioning: the joint effects of parental alcoholism, gender and childhood socio-economic stress. Addiction, 1997, 92 (5), 583-596.

(19) Aragón, N., Bragado, M.C., y Carrasco, I. Alcoholismo parental y psicopatología infantojuvenil. Una revisión. Adicciones, 1997, 9 (2), 255-277.

(20) Pérez-Galvez, B.S., y Vicente, M.P. Trastornos psicopatológicos en hijos de alcohólicos: Diferencias en relación al tiempo de abstinencia paterna y sexo filial. Libro de actas de las XX Jornadas de Socidrogalcohol. Córdoba. Noviembre, 1992

(21) Jiménez, J.L., Barrios, A., Yoller, A.B., y Pérez de Heredia, J.L. Psicopatología en hijos de padres alcohólicos. Revista de Psiquiatría Infantil, 1992, 2, 95-99.

(22) Wegscheider, S. Another chance: Hope and health for the alcoholic family. Palo Alto: Science and Behavior Books. 1981.

(23) Black, C., Bucky, S.F. y Wilder-Padilla, S. The interpersonal and emotional consequences of being an adult child of an alcoholic. International Journal of the Addictions, 1986, 21, 213231.

(24) Harter, S.L. Psychosocial adjustment of adult children of alcoholics: A review of the recent empirical literature. Clinical Psychology Review, 2000, 20 (3), 311-337.

(25) Christiansen, H.B., Bilenberg, N. Behavioural and emotional problems in children of alcoholic mothers and fathers. European Child and Adolescence Psychiatry, 2000, 9, 219-226.

(26) Sher, K.J. Children of alcoholics. A critical appraisal of theory and research. Chicago: University of Chicago Press. 1991. 
(27) Goodwin, D.W. Alcoholism and heredity: A review and hypothesis. Archives of General psychiatry, 1979, 36, 57-61.

(28) Cloninger, C.R. Neurogenetic adaptive mechanisms in alcoholism. Science, 1987, 236, 410416.

(29) Seilhamer, R.A. y Jacob, T. Family factors and adjustment in COAs. En M. Windle y J.S. Searles (Eds.). Children of alcoholics. Critical perspectives. New York: Guildford Press, 1990 (pp. 168-185).

(30) Tarter, R.E., y Vanyukov, M. Alcoholism: A developmental disorder. Journal of Consulting and Clinical Psychology, 1994, 62 (6), 10961170.

(31) Zucker, R.A., Fitzgerald, H.E. y Moses, H.D. Emergence of alcohol problems and the several alcoholism: A developmental perspective on etiologic theory and life course trajectory. En: D. Cicchetti y D. Cohen (Eds.). Manual of Developmental Psychopathology. New York: Wiley. 1995, Vol 2. (pp. 677-711).

(32) Pogge, D.L., Strokes, J., y Harvey, Y. Psychometric vs attentional correlates of early alcohol and substance abuse. Journal of Abnormal Psychology, 1992, 20 (2), 151-162.

(33) Berman, S.M., Whipple, S.C., Fitch, R.J. y Noble, E. P. P3 in young boys as a predictor of adolescent substance abuse. Alcohol, 1993, 10, 69-76.

(34) Schuckit, M.A., y Smith, T.L. An 8-year followup of 450 sons of alcoholic and control subjects. Archives of General Psychiatry, 1996, 53, 202-210.

(35) Volavka, J., Czobor, P., Goodwin, D.W., Gabrielli, W.F., Penick, E.C., Mednick, A., Jensen, P., Knop, J., y Schulsinger, F. The electroencephalogram after alcohol administration in high-risk men and the development of alcohol use disorders 10 years later. Preliminary findings. Archives of General Psychiatry, 1996, 53, 258-263.

(36) Webb, J.A., Baer, P.E., y McKelvey, R.S. Development of a risk profile for intentions to use alcohol among fifth and sixth graders. Journal of the American Academy of Child and Adolescent Psychiatry, 1995, 34 (6), 772778.

(37) Poikolainen, K. Risk factors for alcohol dependence: A questionnaire survey. Alcoholism: Clinical and Experimental Research, 1997, 21 (6), 957-961.
(38) Vitaro, F., Dobkin, P.L., Carbonneau, R., y Tremblay, R.E. Personal and familiar characteristics of resilient sons of male alcoholics. Addiction, 1996, 91 (8), 1161-1177.

(39) Dobkin, P.L., Tremblay, R.E., Desmarais-Gervais, L., y Dépelteau, L. Is having an alcoholic father hazardous for children's physical health? Addiction, 1994, 89, 1619-1628.

(40) Díaz, R., y Gual, A. Adolescent living with parental alcoholism. Alcoscope, 2001, 4(2), 915.

(41) Dobkin, P.L. y Dongier, M. Les parents ont mangé des raisins verts et les enfants n'ont pas eu les dents agacées: peut-on identifier des facteurs de protection contre le risque génétique de devenir alcoolique?. Psychotropes, 1990, V (2), 15-21.

(42) Hill, E.M., Nord, J.L., Blow, F.C. Young-adult children of alcoholic parents: protective effects of positive family functioning. British Journal of Addiction, 1992, 87, 1677-1690.

(43) Wolin, S., y Wolin, S.J. The challenge model. Working with strengths in children of substance-abusing parents. Child and adolescent psychiatric clinics of North America, 1996, 5 (1), 243-256.

(44) Hussong, A.M., y Chassin, L. Substance use initiation among adolescent children of alcoholics: Testing protective factors. Journal of Studies on Alcohol, 1997, 58, 272-279.

(45) Díaz, R. Programa ALFIL: Intervención psicoeducativa para jóvenes con antecedentes familiares de alcoholismo. Manual para educadores y terapeutas. Socidrogalcohol. Anagrafic, S.A. Barcelona. 2000.

(46) Gual, A., y Díaz, R. Children of alcoholics: Risk factors and preventive strategies. WHO Ministerial Conference about Young People and Alcohol. Working group: The Family, Stockholm. Sweden. February, 2001.

(47) Nye, C.L., Fitzgerald, H.E., Zucker, R.A. Early intervention in the path to alcohol problems through conduct problems: treatment involvement and child behavior change. Journal of Consulting and Clinical Psychology, 1995, 63 (5), 831-840.

(48) Dawson, D.A., Harford, T.C. y Grant, B.F. Family history as a predictor of alcohol dependence. Alcoholism: Clinical and Experimental Research, 1992, 16(3), 572-575. 
(49) Hohman, M.M. Motivational Interviewing: An intervention tool for child welfare case workers working with substance abusing parents. Child Welfare, 1998, LXXVII (3), 275-289.

(50) Woodside, M., Coughey, K., y Cohen, R. Medical costs of children of alcoholics: pay now or pay later. Journal of Substance Abuse, 1993, 5 (3), 281-287.

(51) Children of Alcoholics Foundation. Children of Alcoholics in the Medical System: Hidden Problems, Hidden Costs. Children of Alcoholics Foundation, Inc., New York, NY. 1990.

(52) Bates, M.E., y Labouvie, E.W. Adolescent risk factors and the prediction of persistent alcohol and drug use into adulthood. Alcoholism: Clinical and Experimental Research, 1997, 21 (5), 944-950.

(53) Hall, C.W., Bolen, L.M., Webster, R.E. Adjustment issues with adult children of alcoholics. Journal of Clinical Psychology, 1994, 50 (5), 787-791.

(54) Giunta, C.T., y Compas, B.E. Adult daughters of alcoholics: Are they unique?. Journal of Studies on Alcohol, 1994, 55, 600-606.

(55) Roosa, M.W., Gensheimer, L.K., Short, J., Ayers, L.S., and Short, J.L. Development of school-based prevention program for children in alcoholic families. Journal of Primary Prevention, 1990, 11, 119-141.

(56) Short, J.L., Roosa, M.W., Sandler, I.N., Ayers, T.S., Gensheimer, L.K., Braver, S.L., y Yein, J. Evaluation of a preventive intervention for a self-selected subpopulation of children. American Journal of Community Psychology, 1995, 33 (2), 223-247.

(57) Emshoff, J.G. A preventive intervention with children of alcoholics. Prevention in Human services, 1990, 7 (1), 225-253.

(58) Price, A.W., y Emshoff, J.G. Breaking the cycle of addiction. Alcohol, Health and Research World, 1997, 21(3), 241-246.

(59) Kumpfer, K.L., Molraard, V., y Spoth, R. The "Strengthening Families Program" for the prevention of delinquency and drug use. En R. Peters, y R. McMahon (Eds.) Preventing childhood disorder: substance abuse and delinquency. Newburg, CA: Sage Publications. 1996 (pp.241-267).

(60) Davis, R.B., Wolfe, H., Orenstein, A., Bergamo, P., Buetens, K., Fraster, B., Hogan, J., MacLean, A., y Ryan, M. Intervening with high risk youth: A program model. Adolescence, 1994, 29 (116), 763-774. (CASPAR Cambridge and Somerville Program for Alcoholism Rehabilitation. Massachusetts. Alcohol Education program)

(61) Nastasi, B.K., y DeZolt, D. M. School interventions for children of alcoholics. New York: The Guildford Press. 1994

(62) Nastasi, B. K. Groups for prevention and intervention with children of alcoholics. En K. Callan, y T.R. Kratochwill (Eds.) Handbook of group intervention for children and families. Needam Heights, MA: Allyn \& Bacon. 1998 (pp.193-219).

(63) Eurocare y Coface. Alcohol problems in the family. A report to the European Union. European Commission Directorate General V. England. 1998.

(64) Emshoff, J.G. y Price, A.W. Prevention and intervention strategies with children of alcoholics. Pediatrics, 1999, 103 (5), 1112-1121.

(65) Kern, J.C. Management of children of alcoholics. En S. Zinberg, J. Wallace y S. Blume (Eds.). Practical approaches to alcoholism psychotherapy. New York: Plenum Press. 1985.

(66) Seleckman, M. Abrir caminos para el cambio. Soluciones de terapia breve para adolescentes con problemas. Colección Terapia Familiar. Barcelona: Ed. Gedisa. 1996.

(67) Rollnick, S., y Miller, W. What is motivational interviewing?. Behavioural and Cognitive Psychotherapy, 1995, 23 (4), 325-334.

(68) Díaz, R., Gual, A., y Grau, C. Intervención preventiva: Diseño de un programa de reducción del riesgo familiar en hijos de alcohólicos. Libro de ponencias de las XXIV Jornadas Nacionales de Socidrogalcohol. Alicante. Marzo, 1997.

(69) Díaz, R. y Gual, A. Evaluation of psychoeducational sessions for children of alcoholics from the ALFIL Program in Spain. 1st European Workshop on Families of People with Alcohol or Mental Health Problems. Bjärsjölagard's Castle. Sweden. August, 2001.

(70) Díaz, R. Hijos de alcohólicos: ¿debemos intervenir?. La experiencia del Programa ALFIL. XXVI Jornadas Nacionales de Socidrogalcohol. Sta. Cruz de Tenerife. Marzo, 1999. (Resumen en la revista Adicciones, 1999, 11, Supl.1, pp. 14). 
(71) Díaz, R., y Gual, A. ALFIL program: a multicomunity study of children of alcoholics in Spain: Effects of familial density of alcoholism on cognitive measures. Póster en el VII Congress of the European Society for Biomedical Research on Alcoholism (ESBRA). Barcelona. Junio, 1999. (Resumen publicado en Alcohol and Alcoholism, 1999, 34(3), 445).

(72) Kumpfer, K.L. Outcome measures of interventions in the study of children of substance abusing parents. Pediatrics, 1999, 103 (5), 1128-1144.

(73) Socidrogalcohol. ¿Alcohol en la familia? Folleto de autoayuda para familias con problemas de alcohol. Anagrafic, S.A., 2001

(74) Wiers, R.W., Gunning, W.B., Sergeant, J.A. Do young children of alcoholics hold more positive or negative alcohol-related expectancies than controls?. Alcoholism: Clinical and Experimental Research, 1998, 22 (8), 1855-1864. 